\title{
Selected E2F2 Polymorphisms in Oral and Oropharyngeal Squamous Cell Carcinoma
}

\author{
Karolina Gołąbek (D, ${ }^{1}$ Krzysztof Biernacki, ${ }^{1}$ Jadwiga Gaździcka, ${ }^{1}$ Joanna K. Strzelczyk, ${ }^{1}$ \\ Katarzyna Miśkiewicz-Orczyk, ${ }^{2}$ Lukasz Krakowczyk, ${ }^{3}$ Natalia Zięba, ${ }^{2}$ Paweł Kiczmer, ${ }^{4}$ \\ Zofia Ostrowska, ${ }^{1}$ and Maciej Misiolek ${ }^{2}$ \\ ${ }^{1}$ Department of Medical and Molecular Biology, Faculty of Medical Sciences in Zabrze, Medical University of Silesia in Katowice, \\ Zabrze 41-808, Poland \\ ${ }^{2}$ Department of Otorhinolaryngology and Oncological Laryngology, Faculty of Medical Sciences in Zabrze, Medical University of \\ Silesia in Katowice, Zabrze 41-800, Poland \\ ${ }^{3}$ Clinic of Oncological and Reconstructive Surgery, Maria Sklodowska-Curie National Research Institute of Oncology, \\ Gliwice 44-102, Poland \\ ${ }^{4}$ Department of Pathomorphology, Faculty of Medical Sciences in Zabrze, Medical University of Silesia in Katowice, \\ Zabrze 41-800, Poland
}

Correspondence should be addressed to Karolina Gołąbek; kgolabek@sum.edu.pl

Received 15 August 2020; Revised 3 November 2020; Accepted 12 March 2021; Published 30 March 2021

Academic Editor: Kazim Husain

Copyright (c) 2021 Karolina Gołąbek et al. This is an open access article distributed under the Creative Commons Attribution License, which permits unrestricted use, distribution, and reproduction in any medium, provided the original work is properly cited.

\begin{abstract}
Oral squamous cell carcinoma (OSCC) and oropharyngeal squamous cell carcinoma (OPSCC) are subgroups of head and neck squamous cell carcinoma. E2F Transcription Factor 2 (E2F2) could contribute to cancer development, because it plays a critical role in many cellular processes, including the cell cycle, proliferation, differentiation, DNA damage response, and cell death. In the current study, we assessed the associations of five E2F2 polymorphisms (rs6667575, rs3218121, rs3218211, rs3218148, and rs3218203) with OSCC and OPSCC and influence on the TNM staging and grading. This is the first such survey to concern the European population. The study included 94 primary tumour samples following surgical resection from patients, whereas the control group consisted of 99 healthy individuals. We tried a matching of cases and controls for age and sample size. DNA samples were genotyped by employing the $5^{\prime}$ nuclease assay for allelic discrimination. Our results suggested that the most significant difference between the control group and the cancer group was the A/G heterozygote for rs3218121. Samples containing this genotype were mostly found in the control group. In our samples, rs6667575, rs3218121, rs3218211, and rs3218148 polymorphisms may affect the course of OSCC and OPSCC, while rs3218203 was not associated with OSCC and OPSCC. However, further studies are warranted to confirm our findings.
\end{abstract}

\section{Introduction}

Head and neck squamous cell carcinoma (HNSCC) is an epithelial tumour with more than 800000 cases diagnosed each year $[1,2]$ with the overall 5 -year survival rate of approximately $40-50 \%$ [2]. Oral squamous cell carcinoma (OSCC) is the most common type of HNSCC. HNSCC is also common in the oropharynx (OPSCC) [3]. The incidence of these two types of HNSCC is still increasing $[4,5]$. Exposure to tobacco and moderate alcohol consumption are important etiological factors in HNSCC carcinogenesis. Infections with high-risk human papillomaviruses (HPV) are responsible for an increasing proportion of OSCC $[6,7]$. Other factors include poor oral hygiene, exposure to carcinogenic chemicals, and poor diet $[6,8,9]$. Another potential group of risk factors is related to endogenous factors such as genetic predisposition [9]. Single nucleotide polymorphisms (SNPs) are typical examples of this group [10]. Some studies showed 
TABLE 1: E2F2 polymorphisms and primers used in the study.

\begin{tabular}{lc}
\hline SNP ID & Context sequence [VIC/FAM] \\
\hline rs6667575 & ATAAGACCCTTTTACTCTAGTCTAC[A/G]TATCTCATTGGTCCTTTTTGGTCCT \\
rs3218121 & TCTATTCAGCGCCTACAGGATGCCA[A/G]GCACCATGCTAGATCCTTACAAGCG \\
rs3218211 & GAGGCCTAAGTGCAATTAGCATTCT[A/G]GCAGACTGGACAGCCCCTCAGAGTC \\
rs3218148 & GCTCCTCTCCACCCTGTTGCCACCC[A/G]GGCCCCAATTAGGCCCAGAGCTGCA \\
rs3218203 & GTAGCCTCAGCTTGTCTCCACTTCC[C/G]TATTACTATTCTCTCTTCAACTCAC \\
\hline
\end{tabular}

that E2F Transcription Factor 2 (E2F2) promoter polymorphisms, which affect the expression of E2F2, are significantly associated with increased risk of many cancers [11-15]. E2F Transcription Factor 2 (E2F2) could contribute to cancer development, because it plays a critical role in many cellular processes, including the cell cycle, proliferation, differentiation, DNA damage response, and cell death [16-18]. The transcription factors of the E2F family are the dimers of $\mathrm{E} 2 \mathrm{~F}$ and DP proteins. It is known that E2F2 is a member of this transcription factor family and these factors have already been identified to bind to the RB protein. The RB/E2F pathway plays an important role as a regulator of cell proliferation at the G1/S checkpoint. E2F-DP complexes may promote cell entry into the $S$ phase. As long as the E2F-DP complex is inactivated, the cell is stopped in phase G1. When RB binds to E2F, the resulting complex acts as a growth promoter [19].

One study was found which presented the relationship between five E2F2 polymorphisms (rs6667575, rs3218121, rs3218211, rs3218148, and rs3218203) and HNSCC (of different locations) in the population from USA (white, not Latino) [20]. In Europe, we have no data regarding those issues. Because of the expected differences in genotype frequencies between ethnic groups in the current study, we assessed the associations of E2F2 polymorphisms rs6667575, rs3218121, rs3218211, rs3218148, and rs3218203 with the risk of OSCC and OPSCC and influence on the TNM staging and grading in the Polish population.

\section{Materials and Methods}

2.1. Patients and Samples. The study included 94 primary tumour samples obtained from Polish patients following surgical resection at the Department of Otorhinolaryngology and Oncological Laryngology, Faculty of Medical Sciences in Zabrze, Medical University of Silesia in Katowice, and the Maria Sklodowska-Curie National Research Institute of Oncology (formerly known as the Maria Sklodowska-Curie Memorial Cancer Centre and Institute of Oncology), Gliwice, Poland. All tumours collected during surgery were OSCC and OPSCC (following the ICD-10 classification-C01: 42; C04.8-C05.2: 23; C09: 14; C05.8-C06.2: 13; C00.5: 1; and C03.0-C03.1: 1). Tumour staging was based on the American Joint Committee on Cancer (AJCC, version 2007) [21, 22] and WHO Classification of Head and Neck Tumours [23]. All samples were immediately frozen at $-80^{\circ} \mathrm{C}$ until DNA extraction. These tumour samples were histologically confirmed by pathologists and were classified as primary tumours. Patients included in this study had no history of preoperative radio- or chemotherapy. The control group consisted of 99 healthy individuals without a history of cancer at any site or oral precancerous disease. We tried a matching of cases and controls for age and sample size. All patients and controls were Caucasians who lived in Poland. The study was approved by the Bioethics Committee of the Medical University of Silesia (Katowice, Poland; approval no. KNW/022/KB1/49/16 and no. KNW/002/KB1/49/II/16/17) and the Institutional Review Board on Medical Ethics of the Maria Sklodowska-Curie Memorial Cancer Centre and Institute of Oncology in Gliwice (Poland; approval no. KB/493$15 / 08$ and no. KB/430-47/13).

2.2. DNA Extraction and SNP Analyses. The methodology of DNA isolation and SNP analyses was also described for the first time in the previous study [24]. Genomic DNA was extracted from each tumour sample $(20 \mathrm{mg})$ by DNeasy Blood \& Tissue Kits (Qiagen, Hilden, Germany) according to the manufacturer's instructions, after tissue homogenization in a FastPrep ${ }^{\circledR}-24$ instrument using Lysing Matrix A tubes (MP Biomedicals, Solon, CA, USA). In the control group, the DNA was extracted from swabs taken from oral mucous membranes using a Swab-Extract DNA Purification Kit (EURx, Gdansk, Poland), according to the manufacturer's instructions. Next, high-quality DNA was eluted in a low-salt buffer containing $10 \mathrm{mM}$ Tris- $\mathrm{HCl}, \mathrm{pH}$ 8.5. The qualitative and quantitative analysis of all isolated DNA was performed by spectrophotometry in a Biochrom WPA Biowave DNA UV/Vis Spectrophotometer (Biochrom, Cambridge, UK), according to the manufacturer's instructions.

Genotyping was conducted with a QuantStudio 5 RealTime PCR System (Applied Biosystems, Foster City, CA, USA). The reaction solution contained $5 \mu \mathrm{g}$ DNA $(5.5 \mu \mathrm{l})$, $12.5 \mu \mathrm{l}$ TaqMan Genotyping Master Mix (Applied Biosystems, Foster City, CA, USA), and $1.25 \mu \mathrm{l}$ TaqMan Genotyping Assay (Applied Biosystems, Foster City, CA, USA). SNP calling was read out automatically in QuantStudio Design and Analysis Software v1.5.1 (Applied Biosystems, Foster City, CA, USA). The types of polymorphisms and the primers used in the study are listed in Table 1.

2.3. Statistical Analysis. The Hardy-Weinberg equilibrium was used for the entire study group and separately for the control and cancer groups. The Kruskal-Wallis test was used for comparison for gender, age, smoking, and alcohol consumption between the control and patient groups. The Fisher exact test was used to search for selected parameters (gender, smoking, and alcohol consumption) with a significant influence on the cancer odds and genotypes with a significant influence on the cancer risk and TNM staging and grading 
in the cancer cohort. All statistical analyses were performed using Power Analysis Software STATISTICA v. 13.36.0 (StatSoft, Krakow, Poland); $\alpha=0.05$ was used in all tests.

\section{Results}

3.1. Patient Characteristics. A total of 94 patients with OSCC and OPSCC were included in this study. Their clinical parameters are given in Table 2. The average age was 62 years (range: $15-78$ years). There were $68(73 \%)$ men and $26(27 \%)$ women; 65 (68\%) patients were smokers; 64 (67\%) reported alcohol consumption; 51 (54\%) were both smokers and alcohol users. The control group consisted of 99 healthy individuals. The average age of the controls was 52.92 years (range: 18-69 years). This group comprised 22 (22\%) men and 77 (78\%) women, of whom 20 were smokers (20\%), 67 were drinkers (68\%), and 20 were both tobacco and alcohol users (20\%).

3.2. SNP Distribution of E2F2 Polymorphism. The SNP distribution is presented in Table 3. All SNP distributions followed the Hardy-Weinberg equilibrium with the exception of rs3218121. Patients with rs3218121 had significantly fewer heterozygotes than the control group, while the control group had no reference homozygotes, which could be due to the limited number of samples.

3.3. Demographics and Risk Factors for Study Subjects. The most significant factors in our study that could contribute to cancer were gender (females had significantly lower odds of cancer than males, $\mathrm{OR}=9.15, p<0.001$ ) and smoking (smoking increased the odds of cancer, OR $=0.11, p<$ 0.001 ). However, in the case of all parameters (gender, smoking, and alcohol consumption), there was a significant difference between the control and cancer groups (Kruskal-Wallis test, $p<0.001$ for all tested parameters).

3.4. Effects of E2F2 Polymorphism on Odds OSCC and OPSCC. Table 4 shows the associations of each individual polymorphism with odds of OSCC and OPSCC. The most significant differences between the control group and the cancer group were found in the case of the A/G heterozygote for rs3218121. Samples containing this genotype were mostly found in the control group. Therefore, this genotype seemed to be significant in cancer odds reduction $(\mathrm{OR}=127.51, p<0.001)$.

3.5. Associations of E2F2 Genotypes with TNM Staging and Grading in the OSCC and OPSCC and Cohort Groups. We used the Fisher exact test separately in the control and cancer groups, as well as in the combined cohort with the assumption that TNM staging and grading were absent in controls.

The heat map represents the influence of a specific genotype based on the odds of specific TNM staging and grading. Significant values $(p \leq 0.05)$ are presented as $\log _{2}(\mathrm{OR})$, insignificant values $(p>0.05)$ as 0 (no change in odds), and incalculable cases as '-'. Cases that significantly increased the odds of specific TNM staging and grading are marked in red, whereas a significant decrease in the odds is marked in green. Values in each case represent $\log _{2}$ of the Fisher exact odds ratio. Therefore, negative values represented how much
TABLE 2: Clinical parameters of patients with OSCC and OPSCC.

\begin{tabular}{lc}
\hline Clinical parameters & Patients, $n(\%)$ \\
\hline Histological grading & \\
G1 (well differentiated) & $16(17)$ \\
G2 (moderately differentiated) & $64(68)$ \\
G3 (poorly differentiated) & $14(15)$ \\
T classification & \\
T1 & $12(13)$ \\
T2 & $23(24)$ \\
T3 & $22(23)$ \\
T4 & $37(39)$ \\
Nodal status & \\
N0 & $42(45)$ \\
N1 & $24(25)$ \\
N2 & $26(28)$ \\
N3 & $2(2)$ \\
\hline
\end{tabular}

higher the odds of specific TNM staging and grading were for a specific genotype or allele, while positive values represented how much lower the odds of specific TNM staging and grading were for a specific genotype or allele. The heat map is presented in Figure 1.

In this study, rs3218211 is the only polymorphism that showed a consistent increase in the odds of $\mathrm{T}=1$ for the G/G homozygote in both the cancer and cohort groups. There was also a consistent decrease in odds of $\mathrm{T}=1$ for both the $A / G$ heterozygote and A allele. However, in the case of the A/A homozygote, we observed an increase in the odds of $\mathrm{N}=2$ in the whole study cohort, with a corresponding decrease in the odds for the $G$ allele. The impact of $A / G$ for rs3218148 in cancer patients was limited to a decrease in the odds of $\mathrm{G}=2$, which could also be found in the combined cohort. However, in the whole study cohort, an additional increase in the odds of $G=2$ could be observed for the A/A homozygote, and the presence of the $\mathrm{G}$ allele decreased those odds. A similar observation was seen when $\mathrm{T}=4$ was analyzed. A/G rs6667575 showed a consistent increase in the odds of $\mathrm{T}=1$ for the $\mathrm{G} / \mathrm{G}$ homozygote in the whole cohort groups. In the case of cancer patients, only the odds of $\mathrm{T}=$ 1 were significantly higher for the A/A rs3218121, which could be additionally observed in the whole study cohort.

\section{Discussion}

It is known that E2F2 is a member of the E2F transcription factor family and these factors have already been identified to bind to the RB protein. The RB/E2F pathway plays an important role as a regulator of cell proliferation at the G1/S checkpoint [19]. Therefore, it seems warranted to determine the significance of E2F2 polymorphisms in carcinogenesis. To the best of the authors' knowledge, not much research on this issue has been done.

The first study related to this subject was performed by Cunningham et al. [25] who associated polymorphisms of selected genes (e.g., rs3218203 and rs760607 E2F2) with the 
TABLE 3: Distribution of E2F2 polymorphism genotypes in the analyzed patient, controls, and cohort groups.

\begin{tabular}{|c|c|c|c|c|}
\hline SNP ID & Genotypes & OSCC and OPSCC+control, $n$ & OSCC and OPSCC, $n$ & Control, $n$ \\
\hline \multirow{4}{*}{ rs6667575 } & G/G & 84 & 46 & 38 \\
\hline & $\mathrm{A} / \mathrm{G}$ & 89 & 36 & 53 \\
\hline & $\mathrm{A} / \mathrm{A}$ & 18 & 10 & 8 \\
\hline & $p$ value & $\leq 1$ & $\leq 1$ & $\leq 1$ \\
\hline \multirow{4}{*}{ rs3218121 } & $\mathrm{G} / \mathrm{G}$ & 80 & 80 & 0 \\
\hline & $\mathrm{A} / \mathrm{G}$ & 82 & 3 & 79 \\
\hline & $\mathrm{A} / \mathrm{A}$ & 31 & 12 & 19 \\
\hline & $p$ value & $\leq 1$ & $\leq 0.001$ & $\leq 0.001$ \\
\hline \multirow{4}{*}{ rs3218211 } & $\mathrm{A} / \mathrm{A}$ & 51 & 25 & 26 \\
\hline & G/A & 90 & 40 & 50 \\
\hline & $\mathrm{G} / \mathrm{G}$ & 51 & 28 & 23 \\
\hline & $p$ value & $\leq 1$ & $\leq 1$ & $\leq 1$ \\
\hline \multirow{4}{*}{ rs3218148 } & $\mathrm{G} / \mathrm{G}$ & 32 & 15 & 17 \\
\hline & $\mathrm{A} / \mathrm{G}$ & 105 & 48 & 57 \\
\hline & $\mathrm{A} / \mathrm{A}$ & 53 & 30 & 23 \\
\hline & $p$ value & $\leq 1$ & $\leq 1$ & $\leq 1$ \\
\hline \multirow{4}{*}{ rs3218203 } & $\mathrm{C} / \mathrm{C}$ & 128 & 65 & 63 \\
\hline & $\mathrm{G} / \mathrm{C}$ & 62 & 30 & 32 \\
\hline & $\mathrm{G} / \mathrm{G}$ & 2 & 0 & 2 \\
\hline & $p$ value & $\leq 0.1$ & $\leq 1$ & $\leq 1$ \\
\hline
\end{tabular}

risk of ovarian cancer and showed this relationship. Next, Justenhoven et al. [12] found that E2F2 rs760607 promoter polymorphism was not significantly associated with the risk of breast cancer.

Lu et.al [20] analyzed 1096 samples of HNSCC with different locations for three SNPs of E2F1 (rs3213180, rs3213182, and rs3213183) and seven SNPs of E2F2 (rs3218121, rs2742976, rs6667575, rs3218203, rs3218148, rs3218211, and rs3218123) and revealed that any of the E2F1 or E2F2 variants alone might not have a substantial effect on HNSCC risk, but a joint effect of the combined risk genotypes (i.e., rs3213182 AA, rs3213183 GG, rs3213180 GG, rs3218121 GG, rs2742976 GT+TT, rs6667575 GA+AA, rs3218203 CC, rs3218148 AA, rs3218211 CC, and rs3218123 GT+TT) might contribute to the risk of HNSCC, and this increased risk was more pronounced among the younger adults ( $\leq 57$ years old), men, never smokers, never drinkers, individuals with a family history of cancer in the first-degree relatives, and patients with oropharyngeal cancer. In our study, we could not investigate the combined effect of polymorphisms on cancer risk due to the small number of samples. Moreover, in the case of rs3218121, a statistically significant disagreement with the HardyWeinberg equilibrium could be observed in genotype frequency distribution for both cancer patients and the control group.

Thus, it seems that our study is the first one to show the relationship between each of those five polymorphisms of E2F2 (i.e., rs3218121, rs3218211, rs3218203, rs6667575, and rs3218148) and the risk of OSCC and OPSCC in the European population. As expected from other literature [9], the most significant factors in our study that could contribute to cancer were gender (females had significantly lower odds of cancer than males, $\mathrm{OR}=9.15, p<0.001$ ) and smoking (smoking increased the odds of cancer, OR $=0.11, p<$ $0.001)$. Our results suggested that the most significant differences between the control group and the cancer group were found in the case of the A/G heterozygote for rs3218121. Samples containing this genotype were mostly observed in the control group.

rs6667575 showed a potentially significant $(p \leq 0.1)$ decrease in the odds of cancer in the case of subjects with the A/G heterozygote. This observation seemed to be additionally supported by the fact that in the whole study cohort, the odds of $\mathrm{T}=1$ were significantly lower for such subjects, while in subjects with the G/G homozygote the odds were significantly increased. However, such findings were found only in the whole study cohort. Therefore, it is possible that the influence of the G/G homozygote on progression could be of minor importance. In the patient group, however, the presence of A/A homozygote seemed to increase the odds $(\mathrm{T}=4)$.

The A/G heterozygote rs3218121 significantly decreased the odds of cancer $(p<0.001)$. In the case of each TNM staging and grading, the influence of the G/G homozygote could significantly increase the odds of staging and grading, while the A/G heterozygote significantly decreased the odds. However, in our study, there was a significant difference in genotype distribution between the cancer and control groups. Additionally, in the case of cancer patients, only the odds of $\mathrm{T}=1$ were significantly higher for the A/A homozygote, which could be additionally observed in the whole study cohort. Therefore, rs3218121 should be further analyzed in future studies. 
TABLE 4: Associations of E2F2 genotypes with odds of OSCC and OPSCC patients and controls.

\begin{tabular}{|c|c|c|c|c|c|}
\hline SNP ID & Genotypes & OSCC and OPSCC & Controls & $p$ value & OR $(95 \% \mathrm{CI})$ \\
\hline \multirow{5}{*}{ rs6667575 } & G/G & 46 & 38 & $\leq 1$ & $0.62(0.35-1.11)$ \\
\hline & $\mathrm{A} / \mathrm{G}$ & 36 & 53 & $\leq 0.1$ & $1.79(1.01-3.19)$ \\
\hline & $\mathrm{A} / \mathrm{A}$ & 10 & 8 & $\leq 1$ & $0.72(0.27-1.91)$ \\
\hline & Var. (A) & 46 & 61 & $\leq 1$ & $1.61(0.90-2.85)$ \\
\hline & Ref. (G) & 82 & 91 & $\leq 1$ & $1.39(0.52-3.68)$ \\
\hline \multirow{5}{*}{ rs3218121 } & $\mathrm{G} / \mathrm{G}$ & 80 & 0 & - & Absent in control \\
\hline & $\mathrm{A} / \mathrm{G}$ & 3 & 79 & $\leq 0.001$ & $127.51(36.38-446.93)$ \\
\hline & $\mathrm{A} / \mathrm{A}$ & 12 & 19 & $\leq 1$ & $1.66(0.76-3.65)$ \\
\hline & Var. (A) & 15 & 98 & - & G/G homozygote absent in control \\
\hline & Ref. (G) & 83 & 79 & $\leq 1$ & $0.60(0.27-1.32)$ \\
\hline \multirow{5}{*}{ rs3218211 } & $\mathrm{A} / \mathrm{A}$ & 25 & 26 & $\leq 1$ & $0.97(0.51-1.84)$ \\
\hline & $\mathrm{G} / \mathrm{A}$ & 40 & 50 & $\leq 1$ & $1.35(0.77-2.39)$ \\
\hline & $\mathrm{G} / \mathrm{G}$ & 28 & 23 & $\leq 1$ & $0.70(0.37-1.34)$ \\
\hline & Var. (G) & 68 & 73 & $\leq 1$ & $1.03(0.54-1.96)$ \\
\hline & Ref. (A) & 65 & 76 & $\leq 1$ & $1.42(0.75-2.71)$ \\
\hline \multirow{5}{*}{ rs3218148 } & $\mathrm{G} / \mathrm{G}$ & 15 & 17 & $\leq 1$ & $1.11(0.52-2.37)$ \\
\hline & $\mathrm{A} / \mathrm{G}$ & 48 & 57 & $\leq 1$ & $1.34(0.75-2.37)$ \\
\hline & $\mathrm{A} / \mathrm{A}$ & 30 & 23 & $\leq 1$ & $0.65(0.34-1.24)$ \\
\hline & Var. (A) & 78 & 80 & $\leq 1$ & $0.90(0.42-1.94)$ \\
\hline & Ref. (G) & 63 & 74 & $\leq 1$ & $1.53(0.81-2.90)$ \\
\hline \multirow{5}{*}{ rs3218203 } & $\mathrm{C} / \mathrm{C}$ & 65 & 63 & $\leq 1$ & $0.86(0.47-1.56)$ \\
\hline & $\mathrm{G} / \mathrm{C}$ & 30 & 32 & $\leq 1$ & $1.07(0.58-1.95)$ \\
\hline & $\mathrm{G} / \mathrm{G}$ & 0 & 2 & - & Absent in cancer \\
\hline & Var. (G) & 30 & 34 & $\leq 1$ & $1.17(0.64-2.13)$ \\
\hline & Ref. (C) & 95 & 95 & - & G/G homozygote absent in cancer \\
\hline
\end{tabular}

\begin{tabular}{|c|c|c|c|c|c|c|c|c|c|c|c|c|c|c|c|c|c|c|c|c|c|c|}
\hline & & & \multicolumn{5}{|c|}{ rs6667575 } & \multicolumn{5}{|c|}{ rs3218121 } & \multicolumn{5}{|c|}{ rs3218211 } & \multicolumn{5}{|c|}{ rs3218148 } \\
\hline & & & $\mathrm{G} / \mathrm{G}$ & $\mathrm{A} / \mathrm{G}$ & $\mathrm{A} / \mathrm{A}$ & $\begin{array}{l}\text { Var. } \\
\text { (A) }\end{array}$ & $\begin{array}{l}\text { Ref. } \\
\text { (G) }\end{array}$ & $\mathrm{G} / \mathrm{G}$ & $\mathrm{A} / \mathrm{G}$ & $\mathrm{A} / \mathrm{A}$ & $\begin{array}{l}\text { Var. } \\
\text { (A) }\end{array}$ & $\begin{array}{l}\text { Ref. } \\
\text { (G) }\end{array}$ & $\mathrm{G} / \mathrm{G}$ & $\mathrm{A} / \mathrm{G}$ & $\mathrm{A} / \mathrm{A}$ & $\begin{array}{l}\text { Var. } \\
\text { (A) }\end{array}$ & $\begin{array}{l}\text { Ref. } \\
\text { (G) }\end{array}$ & $\mathrm{G} / \mathrm{G}$ & $\mathrm{A} / \mathrm{G}$ & $\mathrm{A} / \mathrm{A}$ & $\begin{array}{l}\text { Var. } \\
\text { (A) }\end{array}$ & $\begin{array}{c}\text { Ref } \\
(\mathrm{G}) \\
\end{array}$ \\
\hline \multirow{10}{*}{ OscC } & \multirow{4}{*}{\begin{tabular}{|l|} 
TNM \\
staging
\end{tabular}} & $\mathrm{T} 1$ & 0.00 & 0.00 & 0.00 & 0.00 & 0.00 & 0.00 & - & -2.04 & 0.00 & 0.00 & -2.57 & 2.32 & 0.00 & 2.57 & 0.00 & 0.00 & 0.00 & 0.00 & 0.00 & 0.00 \\
\hline & & $\mathrm{T} 2$ & 0.00 & 0.00 & & 0.00 & 0.00 & 0.00 & & 0.00 & 0.00 & 0.00 & 0.00 & 0.00 & 0.00 & 0.00 & 0.00 & 0.00 & 0.00 & 0.00 & 0.00 & $0.0 c$ \\
\hline & & $\mathrm{T} 3$ & 0.00 & 0.00 & 0.00 & 0.00 & 0.00 & 0.00 & 0.00 & 0.00 & 0.00 & 0.00 & 0.00 & 0.00 & 0.00 & 0.00 & 0.00 & 0.00 & 0.00 & 0.00 & 0.00 & 0.00 \\
\hline & & $\mathrm{T} 4$ & 0.00 & 0.00 & -2.09 & 0.00 & 2.09 & 0.00 & 0.00 & 0.00 & 0.00 & 0.00 & 0.00 & 0.00 & 0.00 & 0.00 & 0.00 & 0.00 & 0.00 & 0.00 & 0.00 & 0.00 \\
\hline & \multirow{6}{*}{ Grading } & No & 0.00 & 0.00 & 0.00 & 0.00 & 0.00 & 0.00 & 0.00 & 0.00 & 0.00 & 0.00 & 0.00 & 0.00 & 0.00 & 0.00 & 0.00 & 0.00 & 0.00 & 0.00 & 0.00 & 0.00 \\
\hline & & N1 & 0.00 & 0.00 & 0.00 & 0.00 & 0.00 & 0.00 & 0.00 & 0.00 & 0.00 & 0.00 & 0.00 & 0.00 & 0.00 & 0.00 & 0.00 & 0.00 & 0.00 & 0.00 & 0.00 & 0.00 \\
\hline & & $\mathrm{N} 2$ & 0.00 & 0.00 & 0.00 & 0.00 & 0.00 & 0.00 & 0.00 & 0.00 & 0.00 & 0.00 & 0.00 & 0.00 & 0.00 & 0.00 & 0.00 & 0.00 & 0.00 & 0.00 & 0.00 & 0.00 \\
\hline & & G1 & 0.00 & 0.00 & 0.00 & 0.00 & 0.00 & 0.00 & 0.00 & 0.00 & 0.00 & 0.00 & 0.00 & 0.00 & 0.00 & 0.00 & 0.00 & 0.00 & 0.00 & 0.00 & 0.00 & $0.0 c$ \\
\hline & & G2 & 0.00 & 0.00 & 0.00 & 0.00 & 0.00 & 0.00 & 0.00 & 0.00 & 0.00 & 0.00 & 0.00 & 0.00 & 0.00 & 0.00 & 0.00 & 0.00 & 1.32 & 0.00 & 0.00 & 0.00 \\
\hline & & G3 & 0.00 & 0.00 & 0.00 & 0.00 & 0.00 & 0.00 & - & 0.00 & 0.00 & 0.00 & 0.00 & 0.00 & 0.00 & 0.00 & 0.00 & 0.00 & 0.00 & 0.00 & 0.00 & 0.00 \\
\hline \multirow{10}{*}{$\begin{array}{c}\text { Combined } \\
\text { study } \\
\text { cohort }\end{array}$} & \multirow{4}{*}{$\begin{array}{c}\text { TNM } \\
\text { staging }\end{array}$} & $\mathrm{T} 1$ & -2.23 & 2.39 & 0.00 & 2.23 & 0.00 & -1.79 & - & -1.33 & 1.79 & 0.00 & -2.34 & 2.41 & 0.00 & 2.34 & 0.00 & 0.00 & 0.00 & 0.00 & 0.00 & 0.00 \\
\hline & & $\mathrm{T} 2$ & 0.00 & 0.00 & 0.00 & - & 0.00 & -4.30 & - & 0.00 & 4.30 & 0.00 & 0.00 & 0.00 & 0.00 & 0.00 & 0.00 & 0.00 & 0.00 & 0.00 & 0.00 & 0.00 \\
\hline & & $\mathrm{T} 3$ & 0.00 & 0.00 & 0.00 & 0.00 & 0.00 & -2.98 & 3.14 & 0.00 & 2.98 & 0.00 & 0.00 & 0.00 & 0.00 & 0.00 & 0.00 & 0.00 & 0.00 & 0.00 & 0.00 & 0.00 \\
\hline & & $\mathrm{T} 4$ & 0.00 & 1.20 & 0.00 & 0.00 & 0.00 & -3.85 & 5.28 & 0.00 & 3.85 & 0.00 & 0.00 & 0.00 & 0.00 & 0.00 & 0.00 & 0.00 & 0.00 & -1.26 & 0.00 & 1.26 \\
\hline & \multirow{6}{*}{ Grading } & No & 0.00 & 0.00 & 0.00 & 0.00 & 0.00 & -3.35 & 5.62 & 0.00 & 3.35 & 0.00 & 0.00 & 0.00 & 0.00 & 0.00 & 0.00 & 0.00 & 0.00 & 0.00 & 0.00 & 0.00 \\
\hline & & $\mathrm{N} 1$ & 0.00 & 1.42 & 0.00 & 0.00 & 0.00 & -2.94 & 4.56 & 0.00 & 2.94 & 0.00 & 0.00 & 0.00 & 0.00 & 0.00 & 0.00 & 0.00 & 0.00 & 0.00 & 0.00 & 0.00 \\
\hline & & $\mathrm{N} 2$ & 0.00 & 0.00 & 0.00 & 0.00 & 0.00 & -4.49 & 4.48 & 2.38 & 4.49 & 0.00 & 0.00 & 0.00 & -1.44 & 0.00 & 1.44 & 0.00 & 0.00 & 0.00 & 0.00 & $0.0 c$ \\
\hline & & G1 & 0.00 & 0.00 & 0.00 & 0.00 & 0.00 & -2.27 & 3.66 & 0.00 & 2.27 & 0.00 & 0.00 & 0.00 & 0.00 & 0.00 & 0.00 & 0.00 & 0.00 & 0.00 & 0.00 & 0.00 \\
\hline & & G2 & 0.00 & 0.00 & 0.00 & 0.00 & 0.00 & -4.67 & 5.66 & 0.00 & 4.67 & 0.00 & 0.00 & 0.00 & 0.00 & 0.00 & 0.00 & 0.00 & 0.94 & -1.06 & 0.00 & 1.06 \\
\hline & & G3 & 0.00 & 0.00 & 0.00 & 0.00 & 0.00 & -4.44 & - & 0.00 & 4.44 & 0.00 & 0.00 & 0.00 & 0.00 & 0.00 & 0.00 & 0.00 & 0.00 & 0.00 & 0.00 & 0.00 \\
\hline
\end{tabular}

FIGURE 1: Heatmap represents the influence of E2F2 genotypes on the TNM staging and grading in the OSCC and OPSCC and cohort groups.

In this study, rs3218211 can be the only polymorphism that showed a consistent increase in the odds of $\mathrm{T}=1$ for the G/G homozygote in both the cancer and whole cohort groups and a consistent decrease in odds of $\mathrm{T}=1$ for both the A/G heterozygote and A allele. Surprisingly, however, in the case of the A/A homozygote, we observed an increase in 
the odds of $\mathrm{N}=2$ in the whole study cohort, with a corresponding decrease in the odds for the $\mathrm{G}$ allele. This may suggest that in the case of progression, the $A / G$ heterozygote could be more beneficial for patients, but the presence of A/A homozygote (and the lack of the $G$ allele) could unfavourably impact cancer progression (node involvement). Despite the above, rs3218211 did not significantly influence the odds of cancer.

The impact of A/G for rs3218148 in cancer patients was limited to a decrease in the odds of $G=2$, which could also be found in the combined cohort. However, in the whole study cohort, an additional increase in the odds of $G=2$ could be observed for the A/A homozygote, and the presence of the $\mathrm{G}$ allele decreased the odds. A similar observation was seen when $\mathrm{T}=4$ was analyzed. The odds were increased for subjects with the A/A homozygote, while the $G$ allele decreased the odds. This may suggest that in the case of rs3218148 the $G$ allele (and by extension also the heterozygote) could be more beneficial for cancer progression. However, no significant influence of any of rs3218148 genotypes could be seen in the case of cancer.

In our study, rs3218203 had no significant impact on the cancer status.

Our research has limitations that need to be addressed. Firstly, the small sample size has attenuated statistical power. Secondly, since activating E2F2 transcription through its interaction with histone deacetylases (HDACs) can facilitate HPV31 E7 replication [26], it would be reasonable to determine E2F2 expression and HPV in further studies.

\section{Conclusions}

In conclusion, in our samples, rs6667575, rs3218121, rs3218211, and rs3218148 polymorphisms may affect the course of OSCC and OPSCC, while rs3218203 was not associated with OSCC and OPSCC. However, further studies are warranted to confirm our findings.

\section{Data Availability}

The data used to support the findings of this study are available from the corresponding author upon request.

\section{Conflicts of Interest}

The authors declare that there is no conflict of interest regarding the publication of this paper.

\section{Authors' Contributions}

KG was responsible for research concept and design; KMO, $€ K$, and NZ were responsible for collection of tumour samples; JG, KG, and JKS were responsible for collection of control group samples; KG and JG were responsible for DNA extraction; KG was responsible for SNP analyses; KG, KB, and $\mathrm{PK}$ were responsible for data analysis; $\mathrm{KG}$ and $\mathrm{KB}$ were responsible for result interpretation; $K G, K B$, and JG were responsible for writing of the article; JKS, ZO, and $\mathrm{MM}$ were responsible for critical revision of the article. All authors read and approved the final manuscript.

\section{Acknowledgments}

This work was supported by the Ministry of Science and Higher Education (KNW-2-005/N/8/N).

\section{References}

[1] F. Bray, F. J. Ferlay, I. Soerjomataram, R. L. Siegel, L. A. Torre, and A. Jemal, "Global cancer statistics 2018: GLOBOCAN estimates of incidence and mortality worldwide for 36 cancers in 185 countries," CA: a Cancer Journal for Clinicians, vol. 68, no. 6, pp. 394-424, 2018.

[2] M. Canning, G. Guo, M. Yu et al., "Heterogeneity of the head and neck squamous cell carcinoma immune landscape and its impact on immunotherapy," Frontiers in Cell and Development Biology, vol. 7, p. 52, 2019.

[3] A. Argiris, M. V. Karamouzis, D. Raben, and R. L. Ferris, "Head and neck cancer," Lancet, vol. 371, no. 9625, pp. 1695-1709, 2018.

[4] R. L. Siegel, K. D. Miller, and A. Jemal, "Cancer statistics, 2016," CA: a Cancer Journal for Clinicians, vol. 66, no. 1, pp. 7-30, 2016.

[5] A. K. Chaturvedi, W. F. Anderson, J. Lortet-Tieulent et al., "Worldwide trends in incidence rates for oral cavity and oropharyngeal cancers," Journal of Clinical Oncology, vol. 31, no. 36, pp. 4550-4559, 2013.

[6] M. Lacko, B. J. M. Braakhuis, E. M. Sturgis et al., "Genetic susceptibility to head and neck squamous cell carcinoma," International Journal of Radiation Oncology • Biology • Physics, vol. 89 , no. 1, pp. 38-48, 2014

[7] C. R. Leemans, P. J. F. Snijders, and R. H. Brakenhoff, "The molecular landscape of head and neck cancer," Nature Reviews Cancer, vol. 18, no. 5, pp. 269-282, 2018.

[8] J. D. Klein and J. R. Grandis, "The molecular pathogenesis of head and neck cancer," Cancer Biology \& Therapy, vol. 9, no. 1, pp. 1-7, 2014.

[9] N. Vigneswaran and M. D. Williams, "Epidemiologic trends in head and neck cancer and aids in diagnosis," Oral and Maxillofacial Surgery Clinics of North America, vol. 26, no. 2, pp. 123-141, 2014.

[10] K. Shridhar, A. Aggarwal, G. K. Walia et al., "Single nucleotide polymorphisms as markers of genetic susceptibility for oral potentially malignant disorders risk: review of evidence to date," Oral Oncology, vol. 61, pp. 146-151, 2016.

[11] J. Chen, C. J. Etzel, C. I. Amos et al., "Genetic variants in the cell cycle control pathways contribute to early onset colorectal cancer in Lynch syndrome," Cancer Causes \& Control, vol. 20, no. 9, pp. 1769-1777, 2009.

[12] C. Justenhoven, C. B. Pierl, and S. Haas, "Polymorphic loci of E2F2, CCND1, and CCND3 are associated with HER2 status of breast tumors," International Journal of Cancer, vol. 124, no. 9, pp. 2077-2081, 2009.

[13] R. V. Pusapati, R. L. Weaks, R. J. Rounbehler, M. J. McArthur, and D. G. Johnson, "E2F2 suppresses Myc-induced proliferation and tumorigenesis," Molecular Carcinogenesis, vol. 49, no. 2, pp. 152-156, 2010.

[14] L. Chen, J. H. Yu, Z. H. Lu, and W. Zhang, "E2F2 induction in related to cell proliferation and poor prognosis in non-small 
cell lung carcinoma," International Journal of Clinical and Experimental Pathology, vol. 8, no. 9, pp. 10545-10554, 2015.

[15] X. Chen, T. Paranjape, C. Stahlhut et al., "Targeted resequencing of the microRNAome and $3^{\prime}$ UTRome reveals functional germline DNA variants with altered prevalence in epithelial ovarian cancer," Oncogene, vol. 34, no. 16, pp. 2125-2137, 2015.

[16] D. Reimer, S. Sadr, A. Wiedemair et al., "Expression of the E2F family of transcription factors and its clinical relevance in ovarian cancer," Annals of the New York Academy of Sciences, vol. 1091, pp. 270-281, 2016.

[17] D. Reimer, S. Sadr, A. Wiedemair et al., "Clinical relevance of E2F family members in ovarian cancer: an evaluation in a training set of 77 patients," Clinical Cancer Research, vol. 13, no. 1, pp. 144-151, 2007.

[18] D. E. Suzuki, A. M. Nakahata, and O. K. Okamoto, "Knockdown of E2F2 inhibits tumorigenicity but preserves stemness of human embryonic stem cells," Stem Cells and Development, vol. 23, no. 11, pp. 1266-1274, 2014.

[19] F. A. Dick, D. W. Goodrich, J. Sage, and N. J. Dyson, "Noncanonical functions of the RB protein in cancer," Nature Reviews. Cancer, vol. 18, no. 7, pp. 442-451, 2018.

[20] M. Lu, Z. Liu, and H. Yu, "Combined effects of E2F1 and E2F2 polymorphisms on risk and early onset of squamous cell carcinoma of the head and neck," Molecular Carcinogenesis, vol. 51, supplement 1, pp. 132-141, 2012.

[21] M. Brunner, B. C. Ng, M. J. Veness, and J. R. Clark, “Comparison of the AJCC N staging system in mucosal and cutaneous squamous head and neck cancer," Laryngoscope, vol. 124, no. 7, pp. 1598-1602, 2014.

[22] P. C. Rodrigues, M. C. C. Miguel, E. Bagordakis et al., "Clinicopathological prognostic factors of oral tongue squamous cell carcinoma: a retrospective study of 202 cases," International Journal of Oral and Maxillofacial Surgery, vol. 43, no. 7, pp. 795-801, 2014.

[23] A. K. El-Naggar, J. K. C. Chan, J. R. Grandis, T. Takata, and P. J. Slootweg, WHO Classification of Head and Neck Tumours, International Agency for Research on Cancer (IARC), Lyon France, 4th edition, 2017.

[24] J. Gaździcka, K. Gołąbek, J. K. Strzelczyk et al., "Selected CDKN2A and MDM2 polymorphisms in oral cavity cancer," Acta Biochimica Polonica, vol. 67, no. 2, pp. 213-218, 2020.

[25] J. M. Cunningham, R. A. Vierkant, and T. A. Sellers, "Cell cycle genes and ovarian cancer susceptibility: a tagSNP analysis," British Journal of Cancer, vol. 101, no. 8, pp. 1461-1468, 2009.

[26] M. S. Longworth, R. Wilson, and L. A. Laimins, "HPV31 E7 facilitates replication by activating E2F2 transcription through its interaction with HDACs," The EMBO Journal, vol. 24, no. 10, pp. 1821-1830, 2005. 